The construction of public space. The contribution of Law 20,958

\section{La construcción del espacio público}

\section{El aporte de la Ley 20.958}

PALABRAS CLAVE $\cdot$ ESPACIO PÚBLICO $\cdot$ LEY DE
APORTES AL ESPACIO PÚBLICO . PLAN DE INVERSIÓN PLAN DE INVERSION EN INFRAESTRUCTURA PARA LA MOVILIDAD Y ESPACIO PÚBLICO IMPUESTO VERDE · CONTRIBUCIÓN A LA CONSTRUCCIÓN DE ESPACIO PÚBLICO

KEYWORDS - PUBLIC SPACE - LAW OF CONTRIBUTIONS TO PUBLIC SPACE · INVESTMENT PLAN IN INFRASTRUCTURE FOR MOBILITY AND PUBLIC SPACE. CREEN TAX. CONTRIBUTION TO THE CONSTRUCTION OF PUBLIC SPACE
RESUMEN

En Chile, el crecimiento urbano ha generado externalidades que afectan al espacio público tensionando su uso debido al aumento y diversificación de las demandas por movilidad, recreación y esparcimiento, evidenciado las carencias de la regulación que ha permitido una producción focalizada en la construcción de bienes factibles de ser transados, descuidando las necesidades por bienes de uso colectivo, situación que la ley 20.958 de aportes al espacio público intentará remediar a través de un sistema de contribuciones destinadas a apoyar la construcción de espacios que favorecen la cohesión social y que es preciso conocer a fin de identificar alcances, procedimientos y responsabilidades que fundamentan y promueven la aplicación, considerando el estado actual de los territorios y la factibilidad de intervenirlos mediante aspectos normativos, operacionales y culturales asociados a la institucionalidad y a la industria desarrolladora.

\section{ABSTRACT}

In Chile, urban growth has generated externalities that affect public space, stressing its use due to the increase and diversification of the demands for mobility, recreation and leisure, evidencing the deficiencies of the regulation that has allowed a production focused on the construction of feasible goods to be traded, neglecting the needs for goods for collective use, a situation that Law 20,958 on contributions to public space will try to remedy through a system of contributions intended to support the construction of spaces that favor social cohesion, necessary to know, in order to identify scopes, procedures and responsibilities that back and promote its application, considering the current state of the territories and the feasibility of intervening them through normative, operational and cultural aspects associated with the institutional framework and the developer industry.

\author{
MARÍA EUGENIA PALLARÉS · Universidad de Chile · mpallare@uchilefau.cl \\ MIRTHA PALLARÉS TORRES · Universidad de Chile · mipallar@uchile.cl
}

Fecha recepción: 30 de septiembre 2020 · Fecha aceptación: 24 de noviembre 2020

\section{INTRODUCCIÓN}

La iniciativa legal fue presentada al Poder Legislativo el 12 de agosto del año 2012, fue promulgada y publicada en octubre del año 2016 bajo el número 20.958 y calendarizó su aplicación para noviembre del año 2020, ocasión en que se aplicó de manera parcial, debido a que en agosto del 2020 el Ejecutivo solicitó postergar por doce meses la exigencia, las razones del aplazamiento se fundamentaron en las dificultades que han existido para realizar los estudios de flujos que se requieren para establecer indicadores. Independiente de las expectativas que la aplicación de la legislación ha generado en las administraciones, la industria y la ciudadanía la medida de su efecto deberá postergarse, circunstancia que desde una perspectiva positiva debe observarse como tiempo que permitirá evaluar alcances, anticipar potenciales conflictos y quizás lo más importante simular aplicaciones apropiadas.

La Ley tuvo como objetivo rescatar el principio original de la legislación urbana chilena respecto de la colaboración entre la industria desarrolladora que ejecuta proyectos y la necesaria contribución que debe hacer para remediar los efectos que provocan sus intervenciones en el territorio. Esta idea de justicia urbana consideró que los efectos de la producción inmobiliaria deben ser asumidos por los que los generan, argumento en línea con lo señalado en el artículo 70 de la Ley General de
Urbanismo y Construcciones (LGUyC) respecto de la cesión de suelo que deben cumplir los proyectos de nuevas urbanizaciones (loteos) y también los de densificación, y que están destinados a la construcción de calles, plazas y equipamiento, entendiendo los espacios destinados al uso común como complementarios a los proyectos de edificación y solidariamente responsables de la conformación de ciudad. Las cesiones de suelo están reguladas en el Artículo 2.2.5 de la Ordenanza General de Urbanismo y Construcciones y dependen de la densidad de ocupación, con un máximo de $44 \%$ de la superficie distribuido en hasta $10 \%$ para áreas verdes, $4 \%$ para equipamiento municipal y hasta $30 \%$ para vialidad.

Importante declarar que la actualización de los instrumentos de planificación ocurre en el tiempo y solo cuando existe evidencia absoluta de su necesidad. El diseño, promulgación y entrada en vigencia de las modificaciones e inclusiones consideran medios y protocolos que garantizan el conocimiento y certidumbre que aseguran que la aplicación se hará en la medida y forma que la ley promovió, circunstancia que agrega más tiempo, provocando conflictos de pertinencia porque se suele apreciar que la ocasión de aplicación es tardía respecto de los problemas que intenta resolver.

En Chile, durante las últimas décadas las áreas urbanas consolidadas han sido tensionadas por el aumento de la demanda por movilidad, 
generalmente provocada por la densificación y el crecimiento de la tasa de motorización, situación que a contar del año 2001 se ha intentado superar incorporado distinto tipo de medidas destinadas a cautelar la provisión de espacio público, siendo quizás la más importante de las modificaciones normativas la obligatoriedad de los proyectos de desarrollo de evaluar el impacto que provocará su inclusión, por lo que deberán presentar un "Estudio de Impacto sobre el Espacio Público" (DS 59, 2011 ), específicamente un "Estudio de Impacto sobre el Transporte Urbano"(EISTU) en el que se indican las obras de mitigación destinadas a remediar los efectos del proyecto sobre el espacio comunitario con énfasis en la movilidad, medida que debe ser aprobada antes de solicitar el permiso de edificación y ejecutarse con cargo al desarrollador previo a la recepción definitiva del proyecto.

En la ocasión la norma fue considerada una respuesta adecuada al desarrollo y gestión de la ciudad, aunque en el tiempo probablemente por falta de seguimiento del efecto no se detectó que su alcance era mínimo, y que lo que en teoría parecía adecuado tenía una escasa cobertura debido a que las condiciones de aplicación eran laxas o podían ser obviadas, adicionalmente considerar que en el tiempo las circunstancias cambiaron y las formas de habitar el territorio se modificaron alterando las solicitaciones. Según la norma actual, la ejecución del estudio de demandas depende de la cantidad de estacionamientos que incorpora cada proyecto con el límite inferior de 250 para uso residenciales y 150 para otros usos, condición que según estimaciones del Ministerio de Vivienda y Urbanismo (MINVU) afecta al 5\% de los permisos de edificación, evidenciando la parcialidad de la medida que libera de la responsabilidad de contribuir a la construcción de la ciudad a la mayoría de los proyectos, además de que las medidas de mitigación se circunscriben al área de instalación del proyecto, por lo que benefician exclusivamente a su operación y están especialmente focalizadas en la motorización, sin mayor interés por otros modos de movilidad y tampoco por el espacio destinado a la recreación y al esparcimiento.

En este contexto la Ley de Aportes al Espacio Público responde a un deseo transversal que involucra el sentimiento de "justica urbana" debido a que establece contribuciones destinadas a remediar los problemas que provocan los proyectos de construcción en los entornos en los que se instalan y en conformidad a los efectos que provocan. Definidas como "mitigación directa o aporte al espacio público". En el texto de la ley se especificaron alcances, procesos y entidades que participarán en la ejecución de obras y/o en el pago directo de tributos destinados a conservar las dotaciones de los lugares en que los proyectos se localizan asumiendo las cargas de uso que incorporan.

Basada en principios, de universalidad la ley hizo extensiva la obligación de mitigar a todos los proyectos independiente de su origen y tamaño, proporcionalidad que obliga a cada proyecto a aportar en función de las externalidades negativas que provoca sobre el territorio (efectos nocivos para la convivencia urbana) que provoca y predictibilidad que condiciona la operación, intentando corregir con métodos objetivos el cálculo y gestión del silencio, indefinición y discrecionalidad que la caracterizaba.

\section{CONSIDERACIONES, OBJETIVOS Y MÉTODOS}

Desde mediados del siglo XX las externalidades negativas provocadas por la intensidad en el uso del suelo han sido entendidas como un problema económico que afecta el bienestar de los individuos, por lo que se postula que pueden mitigarse con impuestos, subsidios 0 controles (Pigou, 2017), estrategias destinadas a equilibrar el efecto de los periuicios con estímulos que compensen las pérdidas, lo que pudiendo parecer efectivo, no profundiza en las sensibilidades asociadas a las culturas locales, factor indispensable a considerar en el diseño de estrategias de planificación y ordenación del crecimiento y desarrollo urbano, que solo cuando están en sintonía con los modos de hacer particulares pueden trascender (Evans, 2006), se trata de propender al bienestar social que depende de la satisfacción de las necesidades colectivas asociadas a la utilidad que proporcionan los bienes y servicios disponibles para la sociedad (Reyes y Rains, 2014).

En el tiempo, la densificación urbana modifica el paisaje y genera conflictos en el acceso a los bienes públicos, debido a que las mitigaciones son precarias y focalizadas en aspectos generales, sin estimación de las necesidades de los distintos públicos. Las remediaciones resuelven problemas de movilidad asumiendo que el desplazamiento es el principal problema, lo que siendo efectivo requiere de una mayor reflexión, porque los modos y necesidades de traslado varían alterando las conductas y las demandas por espacialidades, obligando a una optimización de la oferta de espacio público, que requiere de caracterización y cuantificación de necesidades, que involucren distintas visiones e incorporen a todos los actores que participan del proceso.

La Política Nacional de Desarrollo Urbano chilena promueve el desarrollo responsable del territorio, reconoce las necesidades y sensibilidades de la población y condiciona los proyectos con parámetros de universalidad, sustentabilidad y calidad, intención formalizada a través de instrumentos legales que actualizan y profundizan los modos de producción de la ciudad. Por lo que la dotación de espacio público adecuado y de calidad es el compromiso de los distintos actores que participan de la construcción de ciudad, por lo que el perfeccionamiento de las normativas que regulan sus características e identificación de acciones que lo afectan requiere de un análisis permanente, destinado a anticipar los impactos y explorar alternativas, procurando equilibrar efecto con remediación.

Actualmente existe consenso de que la producción del espacio público es 
responsabilidad de todos, por lo que contribuir a su materialización también debiera serlo, situación que la ley 20.958 intenta formalizar y que es preciso explicitar, identificando las fortalezas y debilidades de las distintas participaciones y las estrategias factibles de asumir para transformarlas en oportunidades. En ese contexto parece necesario superar la barrera de los acuerdos respecto de los principios y definiciones de la ley, resulta relevante profundizar en las inquietudes que existen respecto de su aplicación y de las estructuras creadas para orientarlas. Particularmente en lo relativo a la reglamentación que condiciona la aplicación respecto de las competencias de los actores que participan en cada uno de los procesos, especialmente de los asociados al sector público que se caracterizan por una desigual disponibilidad de medios y recursos. También de los instrumentos técnicos que condicionan la aplicación en relación con los plazos, los indicadores, formatos de cálculo de los aportes y destino de las contribuciones; recordando que el objetivo principal de la legislación es asegurar la conservación de las condiciones actuales de acceso a las dotaciones que provee el espacio público, sin hacerse cargo del déficit y tampoco de las carencias.

La legislación suele ser reactiva y se instala cuando resulta indispensable, por lo que cada vez que se aprueba una modificación a agregación su implementación y aplicación es urgente. El período de tiempo entre la publicación de la ley y su entrada en vigencia es relativo y depende del tipo de ley, de los efectos que tenga sobre otras leyes y por consiguiente las adecuaciones que requiera y su urgencia. En el caso de la ley 20.958 hubo dos plazos importantes de consignar, el primero señalado para la dictación de los reglamentos que permitieran la operación, que fue de seis meses y luego el de entrada en vigencia de los reglamentos que fue de diez y ocho meses luego de la publicación y toma de razón, fijando para noviembre del año 2020 la aplicación, que según últimas indicaciones se postergará parcialmente por un año.
La aplicación de la Ley 20.958 es un desafío para la exploración de múltiples derivadas que consideran desde la revisión de los distintos instrumentos y sus alcances hasta las opciones de instalación en escenarios singulares tensionados por demandas diversas y cambiantes, que reportarán distintas agregaciones que reportarán atributos cualitativos y cuantitativos que mejorarán los entornos y los aspectos asociados a la administración y operación de los recursos que recibirán las administraciones, en las que una de las mayores sensibilidades será la aplicación diferida de la ley, porque generará ingresos antes de conocerse el destino, provocando incertidumbre y desmotivación respecto de la contribución al entenderla como impuesto.

El estudio fue financiado por el Ministerio de Vivienda y Urbanismo y la Facultad de Arquitectura y Urbanismo de la Universidad de Chile (Fondo MINVU para Investigación dirigida a -Promover el desarrollo, la modernización y le progreso de la ciudad-, consideró un plazo de 18 meses y en le desarrollo se estimó pertinente identificar las externalidades que han provocado el crecimiento por densificación y las demandas por espacio público en zonas urbanas consolidadas, intentando caracterizar opciones de mitigación y de producción. Para la ejecución del estudio se diseñó una metodología de carácter exploratorio destinada a recabar antecedentes e información y experiencias que permitieran conocer los alcances y efectos derivados de la aplicación. Los pasos metodológicos utilizados fueron:

Recolección de Información destinada a conocer motivaciones, principios rectores y evolución de la iniciativa legal desde su presentación hasta la promulgación y publicación. Se recurrió a la Biblioteca del Congreso Nacional y a las publicaciones del Diario Oficial.

Trabajo de Campo destinado a identificar desde los territorios la situación respecto de la aplicación de la normativa y de los potenciales aportes que reportará. Se consultó directamente por el estado de avance de la construcción de los instrumentos que la ley y los reglamentos indicaron. El área de estudio escogida fue la que reporta mayor cantidad de permisos de edificación, la plataforma utilizada fue Transparencia Activa y Terreno.

Trabajo de Gabinete destinado a enfrentar la información recabada con la levantada y establecer alternativas de análisis que permitieron construir resultados, identificando las zonas grises de la próxima aplicación y sus efectos sobre la valoración de la iniciativa legal. Adicionalmente también se relevaron potenciales acciones convocantes.

\section{DISCUSIÓN DE RESULTADOS}

La Ley 20.958 impactó distintos instrumentos legales, los que fueron abordados por área de competencia en cuatro artículos. En el Artículo 1 se incluyeron los aspectos que afectaron a la Ley General de Urbanismo y Construcción (MINVU 458, 1975), consideró especificación y agregación de conceptos y términos, además de la inclusión de principios rectores, alcances, participaciones, procesos, obligaciones y responsabilidades, procedimientos y penalizaciones. En el Artículo 2 se incorporaron aspectos relacionados con la Ley 18.696 que había sido modificada por el artículo $6^{\circ}$ de la Ley 18.502 (transporte de pasajeros) abordándose las participaciones en el diseño de informes de mitigación. En el Artículo 3 se establecieron incorporaciones a la Ley 18.695 Orgánica Constitucional de Municipalidades destinados a regular la operatividad y aplicación, estableciendo áreas de competencia, responsabilidades, administración de recursos y plazos de acción. El artículo 4 abordó las modificaciones a la ley 19.175 Orgánica Constitucional sobre Gobierno y Administración Regional, incluyó las modificaciones referidas a la operatividad de los planes de inversión de áreas metropolitanas que cuentan con plan regulador metropolitano o territorios que disponen de planes intercomunales. 
En las Disposiciones Transitorias se abordaron los aspectos operativos de la ley y los tiempos en que se deberán realizar las distintas partidas que se requieren para la entrada en vigencia. Los plazos establecidos fueron de dieciocho meses a constar de la publicación en el Diario Oficial, período en el que los municipios deberán desarrollar y aprobar los Planes de Inversión en Infraestructura para la Movilidad y Espacio Público (PIIMEP) instrumento destinado a proveer de las mejores opciones de movilidad y espacio público a los distintos territorios, materializados en una cartera priorizada de proyectos en sintonía con los instrumentos de planificación local (Plan Regulador Comunal y excepcionalmente Plan de Desarrollo Comunal) que transformarán las ciudades, permitiéndoles superar carencias e incrementar fortalezas desde la singularidad de sus requerimientos.

El reglamento se promulgó el 12 de abril del 2017 mediante los Decretos Supremos $N^{\circ}$ 14 del Ministerio de Vivienda y Urbanismo "Modifica Decreto Supremo Na 47, de Vivienda y Urbanismo de 1992, Ordenanza General de Urbanismo y Construcciones en el sentido de actualizar sus normas a las disposiciones de la ley $N^{a} 20.958$, relativa a aportes al espacio público", y el № 30 del Ministerio de Transporte y Telecomunicaciones - Subsecretaria de Transporte con el "Reglamento sobre mitigación de impactos al sistema de movilidad local derivados de proyectos de crecimiento urbano". El proceso que siguieron los decretos consideró consulta pública e ingreso a Contraloría General de la República para control de legalidad, toma de razón y posterior publicación, importante señalar que durante este proceso lo decretos pueden ser retirados y vueltos a ingresar, procedimiento que no se encuentra normado por lo que se puede extender sin límite de tiempo, cuestión que en este caso ocurrió, publicándose el DS № 14 de aplicación inmediata el 22 de febrero del 2018 y el DS $N^{a}$ 30 de aplicación diez y ocho meses después el 17 de mayo del 2019, fijando como fecha de exigencia el 17 de noviembre del 2020.
En la revisión de la ley se identificaron alcances respecto del área y circunstancias en las que aplica, el modo como lo hace, los actores que participarán y las competencias que tendrán, específicamente las asociadas al sector público representado por los Ministerios involucrados, las Seremi respectivas y las Municipalidades, además del sector desarrollador. La ley instaló instrumentos y conceptos, siendo probablemente los más importantes los asociados a las obligaciones que se localizaron en distintas fronteras, por una parte para el sector público que deberá proveer a la comunidad del "Plan de inversiones en infraestructura de movilidad y espacio público (PIIMEP)", instrumento de nivel comunal o intercomunal que establece una cartera priorizada de proyectos, obras y medidas incluidas en los instrumentos de planificación, corresponde a los deseos que las comunidades anhelan para sus territorios y están destinados a satisfacer las demandas por espacios de encuentro, esparcimiento, recreación, conectividad, accesibilidad, operación y movilidad, contribuyendo a la cohesión social y sustentabilidad urbana, considera los distintos atributos y requerimientos que caracterizan al espacio público y que facilitan la operación de los territorios desde sus singularidades.

Respecto de los desarrolladores, la ley estableció la obligatoriedad de contribución de todos los proyectos que generan densificación en dos modos, por una parte, a través de los "Aportes al Espacio Público" entendido como el tributo que harán los proyectos, pudiéndose materializar en dinero o en la ejecución de estudios, proyectos y obras por un monto equivalente al avalúo fiscal del porcentaje de terreno que se deberá ceder al municipio en que se localiza el proyecto. Además de las "Mitigaciones directas" que corresponde a las medidas y obras que deberán ejecutar dependiendo del impacto que provoquen en la movilidad local, según lo que establezca el respectivo Informe de Mitigación de Impacto Vial (IMIV), entendiendo por "Movilidad" los desplazamientos realizados por modos motorizados o no motorizados que provoca el acceso a actividades y lugares.
Importante destacar que la cuantía de la contribución varía en función del tipo de proyecto, la carga de uso o los usuarios que incorpora y el lugar en que se instala, para el cálculo del aporte se considera el equivalente al $44 \%$ de cesión de suelo que entregan los proyectos de crecimiento por extensión, con un aporte máximo del $44 \%$ del avalúo fiscal del suelo para los proyectos que incorporan densidades superiores a 8.000 habitantes por hectárea, cifra difícil de alcanzar porque actualmente son muy pocos los planes reguladores que en determinadas zonas permiten esa densidad, la contribución promedio en las zonas de mayor densidad de ocupación alcanzan un porcentaje de alrededor del $22 \%$. Respecto de la contribución asociada a las mitigaciones directas y que se materializan en obras de construcción, su cuantía varía en función del lugar en que se localiza el proyecto y fundamentalmente de las opciones que el territorio ofrece para acoger los flujos que se agregarán en los distintos modos. La cuantificación y especificación de las obras a realizar dependen de los flujos vehiculares y los viajes que genere el proyecto, los que se categorizan para determinar el volumen de impacto y las remediaciones que requiere. La determinación del área de influencia para la inducción de flujos es el parámetro más relevante y establece la cantidad de intersecciones que se deben considerar en la producción de mitigaciones, las que deben asegurar el desplazamiento y operación segura para peatones y todo tipo de vehículos motorizados o no.

A fin de conocer el estado de avance en la producción de los planes de inversión que orientarán el destino de los recursos que se recaudarán, se consultó a las comunas de la Región Metropolitana y una comuna por cada región del país (total 67 comunas) evidenciándose el rezago que existe respecto de la disponibilidad del principal instrumento para la administración de la ley, validando la presunción de que se trata de un tema complejo de asumir por las administraciones y confirmando la hipótesis de que probablemente 
será una de las dificultades que tendrá la aplicación. A marzo del año 2020 (8 meses para la entrada en vigencia y tiempo en el que presumiblemente se puede realizar el plan) solo la comuna de Valdivia tenía expuesto en su plataforma de consulta ciudadana la propuesta de PIIMEP que se había adjudicado a través de licitación pública en diciembre del 2018. Alrededor del $30 \%$ de las comunas consultadas declaró que estaban realizando el plan y esperaban promulgarlo para la ocasión en que se requería, el 33\% estaban construyendo insumos para la elaboración del plan, lo que les permitiría concluirlo en un futuro próximo, llamó la atención que el $4,48 \%$ de las comunas declaran no tener relación con el tema y el resto no tener avances, reflejando lo que suele ocurrir en el país respecto de la ocasión de asumir los cambios normativos y que se relaciona con una debilidad sistémica respecto de la disponibilidad de estructuras públicas facilitadoras de la gestión y la gobernanza.

Las dificultades que presenta el diseño del instrumento dicen relación con la amplitud de variables que se deben considerar y las distintas tensiones que es preciso equilibrar, por una parte, las demandas de movilidad y por otra las de esparcimiento y recreación, que se resuelven en un mismo espacio y son reguladas por dos institucionalidades (MINVU y MTT) y dos reglamentos. Históricamente el espacio público ha acogido la funcionalidad asociada al desplazamiento asumiendo el modo como se originaron los asentamientos y las condiciones que la geografía les permitió, generando formas urbanas singulares, resultado de "oposiciones y sustituciones" (Venturini, 1999, 16) erigidas en el tiempo. Los cambios en el uso del espacio público provocado por la evolución de las sociedades, ha transformado los requerimientos, incorporando y desestimado demandas, siendo el más significativo la inclusión del intercambio comercial, que modificó las características de los traslados e instaló actividades de permanencia que facilitaron el encuentro, complejizando la operación, demandando distintas superficies y localizaciones para acoger las múltiples actividades urbanas y articular la sociabilidad (Bladimir, 2005), escenario en el que las administraciones debieron asumir la organización y la producción, a fin de asegurar para la comunidad la posibilidad de relacionarse libremente y desde el anonimato con personas desconocidas generando una integración parcial por objetivos (Schalk, 2007), evidenciando que los asentamientos se caracterizan por un modo de organización espacial en que se liberan áreas para acoger las necesidades colectivas.

El espacio público es el lugar para las actividades colectivas, es donde se instala la oferta de bienes y servicios de libre acceso, posibilitando el desplazamiento y el encuentro, morfológicamente son conectores y nodos, espacialmente están conformados por las estructuras del paisaje y por los espacios construidos, se materializan independiente de objetivos transversales a las localizaciones, son singulares porque en su arquitectura evidencian la identidad local, condicionan la evolución temporal que se materializa con el aporte de distintas generaciones que lo adecuan en función de sueños, circunstancias, necesidades, usos y deseos. Son relevantes para las zonas urbanas porque en ellos se producen las relaciones entre los ciudadanos que la habitan (Borja \& Muxi, 2003), es el lugar desde el que se privilegia el bien común, por lo que deben ser adaptables, acogedores y adecuados a los requerimientos de la población a la que sirven, enfatizando las necesidades colectivas que superan los deseos individuales y/o cotidianos, proveen lugares "para los flujos y lugares" (Ramírez, 2015), destacando la disponibilidad para las prácticas conmemorativas, recreativas, deportivas y contemplativas, y todas las que son parte del ideario de la vida, deben poder adecuarse a fin de acoger y resolver necesidades, ser capaces de convertirse en el contenedor de las inquietudes de los que lo usan, permitiéndoles adaptarlos a sus deseos, considerando los cambios políticos, económicos, sociales y culturales.

Para las ciencias sociales el espacio público es el lugar donde las personas concurren y comparten sus diferencias, para la esfera de lo público es el lugar donde se dialoga con el otro, donde todos pueden ser vistos y oídos (Arendt, 1993), sociológicamente es el de uso público (Schlack, 2007), legalmente es el de propiedad pública, pertenece al Estado y en la legislación chilena se describe como bien nacional de uso público. Las formas que adquiere son diversas y aseguran el desplazamiento de personas y bienes, facilitan la relación de los individuos que participan de la comunidad, son usados de manera transitoria, formalmente se reconocen como áreas abiertas destacándose la calle, la plaza, el parque y los lugares de encuentro ciudadano, son los que acogen la socialización e integración de los distintos grupos etarios, sociales y culturales, son los que permiten conocer la organización de un lugar (Ghel, 2014), son transversales respecto de sus atributos enfocados en las necesidades colectivas.

En el diseño de los planes de inversión (PIIMEP) se deben considerar todos los aspectos que acoge la caracterización, funcionalidades, expectativas y anhelos que las sociedades le asignan al espacio público, actualmente medios y cauces para los distintos flujos en sus diferentes formas, medios, tiempos y velocidades, priorizando las estrategias que promueven y aseguran la visibilidad, movilidad, habitabilidad, seguridad evitando la accidentabilidad, agregando lugares para la relajación, contemplación y distracción mediante la incorporación de estructuras que facilitan el acceso a servicios de bajo impacto que promueven la relación de las personas entre las edificaciones (Ghel, 2006).

En este contexto, la singularidad geográfica es un insumo relevante debido a que sus características suelen ser orientadoras de la morfología urbana y de las alternativas de crecimiento y conectividad con otros territorios. El paisaje condiciona la ubicación, morfología y crecimiento de las comunas, por lo que una selección de territorios espacialmente diversos fue la decisión que se adoptó para analizar escenarios urbanos en los que se deberá proponer una cartera de proyectos apropiados al potencial incremento de uso, a las demandas 


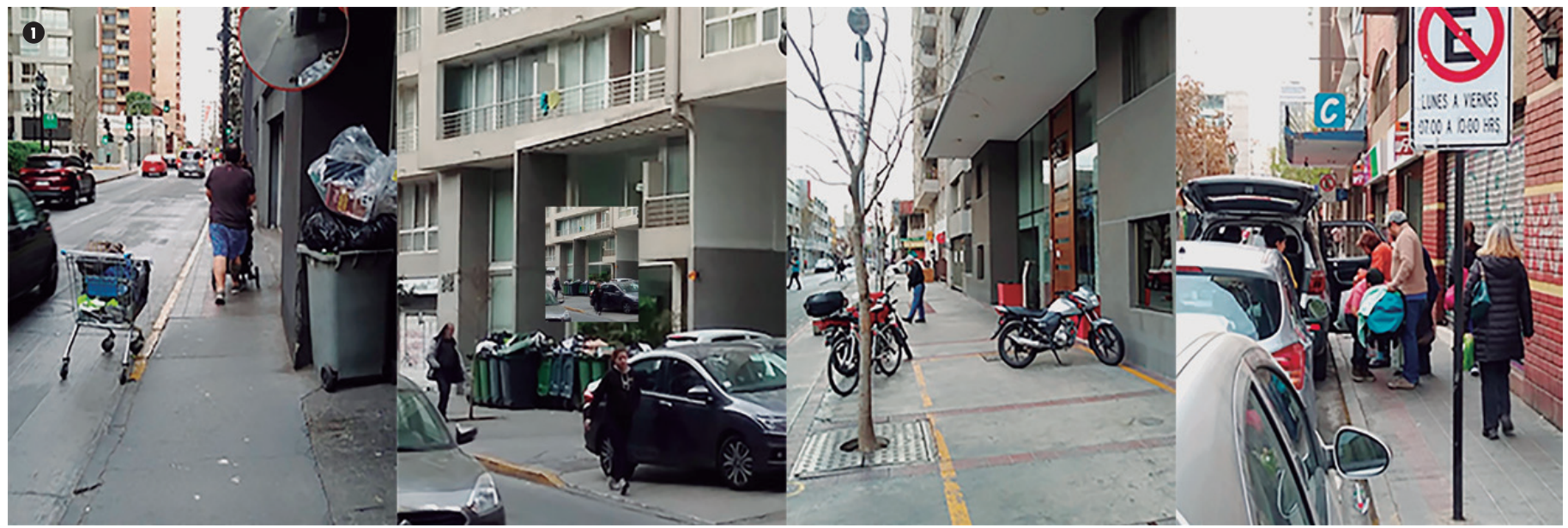

por infraestructura y a espacios para el esparcimiento y la recreación. Intentando cubrir el norte, centro y sur del país, se escogieron seis comunas morfológicamente distintas en las que los límites administrativos tuvieran una impronta paisajística y una producción inmobiliaria significativa. Bajo estos parámetros las comunas escogidas fueron lquique, Antofagasta,

Valparaíso, Viña del Mar, Concepción y Temuco; respecto de la Región Metropolitana y a fin de avanzar en la identificación de situaciones específicas se escogió la zona sur oriente de la comuna de Santiago que se caracteriza por tener la mayor producción inmobiliaria por unidad de superficie, lo que permitirá aproximarse a una zona con diversidad de conflictos, demandas y oportunidades.

En todos los casos se observó que las normas urbanísticas establecidas en los instrumentos de planificación son orientadores de la producción y que en todas las comunas independiente de su tamaño la producción se localiza en las zonas que permiten mayor constructibilidad y mayor densidad, lugares en los que existe mayor cantidad y diversidad de demandas por espacio público y en las que es posible recaudar mayor cantidad de recursos, sin embargo, y por ser las zonas más densificadas los montos factibles de percibir con la aplicación de la ley son bajos, independiente de que los valores de avalúo fiscal son altos, lo que indica que la recaudación representa una contribución que en las zonas de mayor conflicto no resolverán las dificultades actuales y solo contribuirán parcialmente en la medida en que se generen acciones destinadas a sumar aportes.

La revisión de los territorios permitió conocer las externalidades que se deben corregir, y que obedecen a la escasa provisión que existe para espacios destinados a servicios y requerimientos que demandan los ciudadanos y que suelen observarse como lugares comunes (FIGURA 1). Adicionalmente agregar que se detectó que la operación de los territorios cumple un rol relevante en la conservación y administración del espacio público, por lo que debiera asegurarse una financiación que asegure la disponibilidad.

Importante destacar las contribuciones que en el tiempo han hecho los desarrolladores (FIGURA 2), probablemente fuera de norma y focalizadas en la comercialización, independiente de que resulta relevante destacar acciones que sin ser parte de una normativa han contribuido a la creación de espacio público, argumento que desde la administración territorial debiera explotarse, porque los argumentos de venta privada valoran la provisión de espacio público, por lo que es necesario el trabajo conjunto que valora las aportaciones privadas a la producción del espacio público (Figura 3).

\section{REFLEXIONES FINALES}

La ley 20.958 de aporte al espacio público constituye un avance respecto de los instrumentos disponibles para la gestión y administración de las superficies a los que concurren todos los habitantes y usuarios del territorio sin restricción de acceso y goce. Focalizado en la provisión de infraestructuras para la movilidad en distintos modos y espacios para la recreación y el esparcimiento, la ley que se aplicará parcialmente ocho años y tres meses después de haber sido propuesta, constituye una oportunidad para la institucionalidad que podrá desarrollar una planificación urbana integrada destinada a repensar sus dominios e instalar opciones de diseño y gestión que superen las dificultades que actualmente conflictúan la operación de los lugares de todos. Para los desarrolladores, e independiente de la frontera de la que provengan (público o privado) las aportaciones que generan desembolsos económicos se suelen calificar como impuesto a la producción, sin embargo y dadas las modalidades como se estructuraron los 

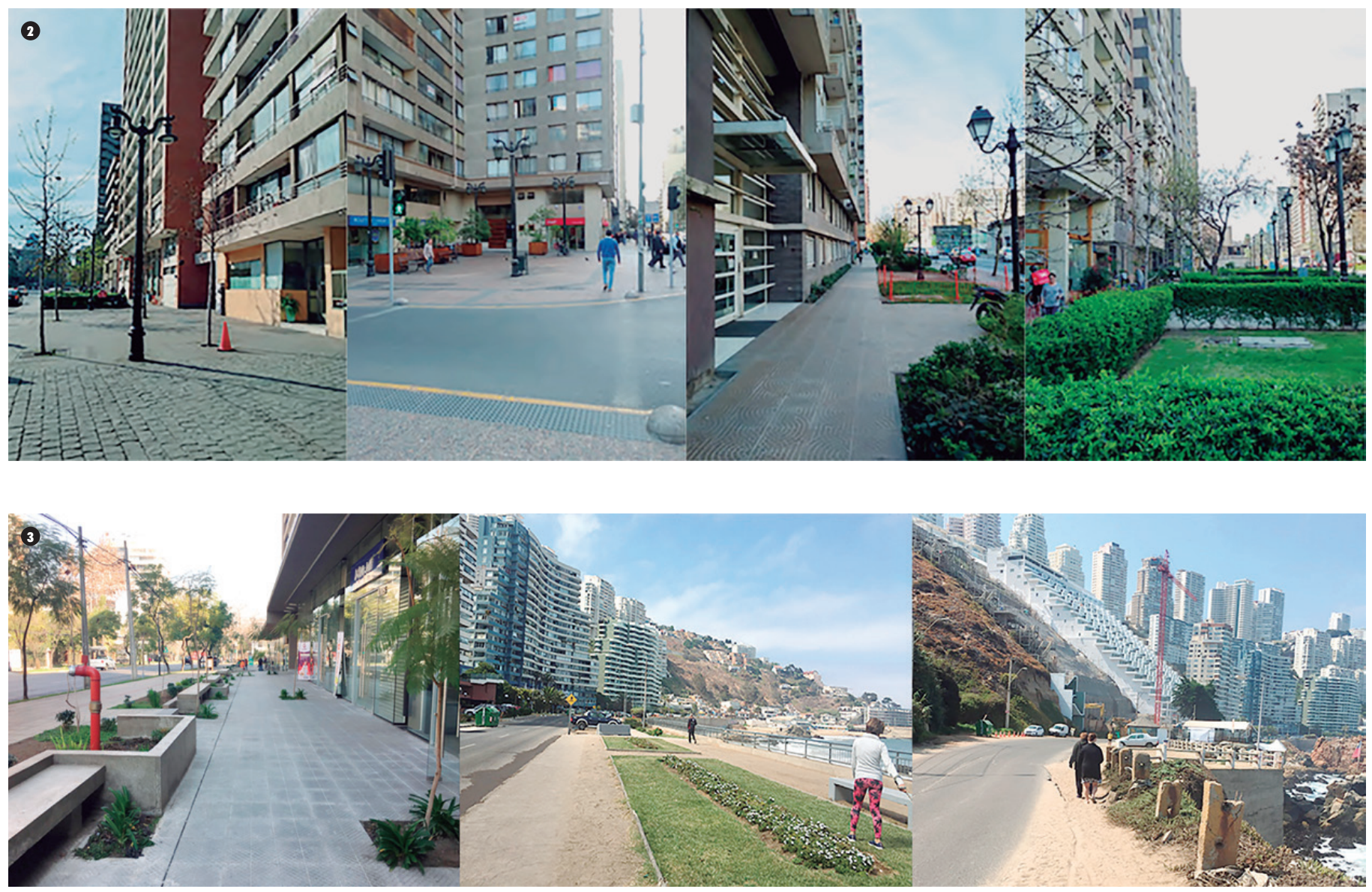

aportes (directo e indirecto) pueden entenderse como una contribución que incorpora un sentimiento de justicia urbana que hace que el desarrollador asuma en parte la mitigación a las externalidades que provoca, haciéndolo solidario en la conservación de los atributos del espacio público y socialmente responsable de sus acciones.

Actualmente solo el $5 \%$ de los proyectos contribuyen en la producción de mitigaciones a las externalidades que generan y de acuerdo al modelo de evaluación lo hacen exclusivamente en el área de instalación del proyecto, a contar de noviembre del año 2020 todos los proyectos contribuirán con aportes destinados a la construcción de espacio público en montos variables dependiendo de la densificación o carga de uso que aportan y del lugar en que se localizan y que condiciona el valor de avalúo fiscal del suelo. Un año después casi todos los proyectos agregaran un aporte en obras destinadas a asegurar las condiciones de movilidad en un área definida por los flujos inducidos por el proyecto en los distintos modos, se excluyen proyectos que generan una escasa cantidad de viajes y movimiento de vehículos. La cuantificación de los aportes depende de cada proyecto y para la mayoría generará un desembolso no previsto que puede transformarse en inversión en la medida en que las administraciones del territorio sean eficientes en la operación y los desarrolladores se anticipen transformando el gasto en inversión. 


\section{REFERENCIAS BIBLIOGRÁFICAS}

Arendt, H. (1993). La Condición Humana. Barcelona, Paidós

Bladimir, G. (2005). La historia de la ciudad es la de sus espacios públicos. Arquitectura y Urbanismo 23 (1), 7-15. Instituto Superior Politécnico José Antonio Echeverría, Ciudad de La Habana, Cuba.

Borja, J., Muxi, Z. (2003). El espacio público, ciudad y ciudadanía. Electa. Barcelona.

Evans, A. (2006). Externalidades, planificación y decisiones colectivas. Santiago. Donde estamos y hacia dónde vamos, CEPAL, 277-297.

Ghel, J. (2014). Ciudades para la gente. Ediciones Infinito. Buenos Aires.

Ghel, J. (2006). La humanización del espacio urbano. La vida social entre los edificios. Editorial Reverté. Barcelona.
Pigou, A.C. (2017). La economía del bienestar. Editorial Aranzadi, Pamplona.

Ramírez, P. (2015). Espacio público, Żespacio de todos? Reflexiones desde la ciudad de México. Revista mexicana de sociología, 77(1), 07-36. Recuperado en 07 de marzo de 2020, de http:// www.scielo.org.mx/scielo.php? script =sci arttext\&pid=S0188-25032015000100001\&lng $=\mathrm{es} \&$ tlng $=\mathrm{es}$.

Reyes, O., Rains, O. (2014). Teoría del bienestar y el óptimo de Pareto como problemas Microeconómicos. Revista Electrónica de Investigación en Ciencias Económicas, Abriendo Camino al Conocimiento, Facultad de Ciencias Económicas, UNAN-Managua. 2(3), 17-34.

file://C:/Users/Maria\%20Eugenia/Downloads/ Dialnet-TeoriaDelBienestarYEIOptimoDeParetoCo moProblemasMi-5109420.pdf
Schlack, E., Vicuña, M. (2011). Componentes normativas de alta incidencia en la nueva morfología del Santiago Metropolitano: una revisión crítica de la norma de "Conjunto Armónico". EURE (Santiago), 37(1 1 1), 131 166. https://dx.doi.org/10.4067/S025071612011000200006

Schlack, E. (2007). Espacio público. ARQ (Santiago). Santiago, 65, 25-27. Disponible en <https:// scielo.conicyt.cl/scielo.php? script $=$ sci arttext\&pid $=$ S0717-69962007000100006\&ln $\mathrm{g}=\mathrm{es} \& \mathrm{nrm}=\mathrm{iso}>$. accedido en 31 marzo 2020. http://dx.doi.org/10.4067/S0717 69962007000100006.

Venturini, E. (1999). La ciudad actual y la necesidad de recuperar las dimensiones humanas de los hechos urbanos. Arquitectura y Urbanismo, No. 4, ISPJAE, La Habana. 